\title{
Subtle Crucial X-Ray Findings in Pediatric Foreign Body Aspiration
}

\author{
Akinori Sekioka $^{1}$, Masashi Koyama ${ }^{2}$, Koji Fukumoto ${ }^{1}$, Akiyoshi Nomura ${ }^{3}$, Naoto Urushihara ${ }^{3}$ \\ 1. Pediatric Surgery, Shizuoka Children's Hospital, Shizuoka, JPN 2. Radiology, Shizuoka Children's Hospital, Shizuoka, \\ JPN 3. Pediatric Surgery, Shizuoka Children's Hospital, Shizuoka, JPN
}

Corresponding author: Akinori Sekioka, akinori-sekioka@noe.saiseikai.or.jp

\begin{abstract}
Foreign body aspiration (FBA), with potentially life-threatening outcomes, is not unusual in the pediatric population. We report two cases of lobar bronchial radiolucent foreign bodies. Chest X-ray (CXR) showed a slight but significant finding of lobar emphysema without a significant mediastinal shift. This is possibly a key to suspecting foreign bodies. In the clinical field, a stepwise approach to detecting foreign bodies is commonly performed, from less invasive options such as CXR to computed tomography (CT). In this context, clinicians should scrupulously check CXRs when pediatric patients complain of respiratory symptoms,
\end{abstract} especially with potential FBA history.

Review began 04/24/2021 Review ended 05/02/2021 Published 05/08/2021

๑) Copyright 2021

Sekioka et al. This is an open access article distributed under the terms of the Creative Commons Attribution License CC-BY 4.0., which permits unrestricted use, distribution, and reproduction in any medium, provided the original author and source are credited.
Categories: Pediatrics, Pediatric Surgery, Radiology

Keywords: children, foreign body, lobar bronchus, radiolucent, lobar emphysema

\section{Introduction}

Foreign body aspiration (FBA) is a relatively common but life-threatening accident in the pediatric population [1,2]. If the detection of FBA is delayed, surgical intervention may be required or, in the worstcase scenario, deadly conditions may ensue [3,4]. A definitive diagnosis of FBA is often challenging because a history of aspiration, physical examination, and radiographical investigations are frequently less evident $[3,5,6]$.

A chest X-ray (CXR) is a basic test for investigating FBA. Although its efficacy is still controversial, it is thought to play an important role as an initial part of the stepwise approach to detecting a foreign body [5,710].

This report describes two cases of FBA with unspecified symptoms and unwitnessed aspiration. CXR findings provided initial clues to the diagnosis and were vital in further evaluation and management.

\section{Case Presentation}

\section{Case 1}

An 11-month-old girl was suspected of ingesting a part of a plastic toy. Because of parental concerns, she was taken to a private clinic the next morning with a mild wheeze. Although her general condition was good and percutaneous oxygen saturation was $98 \%$ (on room air), she was referred to a secondary referral hospital for further evaluation. A CXR showed trivial but concerning right lower lobar emphysema (Figures 1A, 1B). A subsequent chest computed tomography (CT) revealed a foreign body in the right lower lobar bronchus (Figures $1 C, 1 D$ ). Based on these results, she was transferred to our tertiary hospital for foreign body removal. Flexible bronchoscopy was emergently performed under general anesthesia. Open surgery was avoided by use of alligator forceps to remove the plastic foreign body. On postoperative day (POD) 1, her respiratory status and CXR were normal (Figure $1 E$ ) and she was discharged from the hospital. 


\section{Cureus}

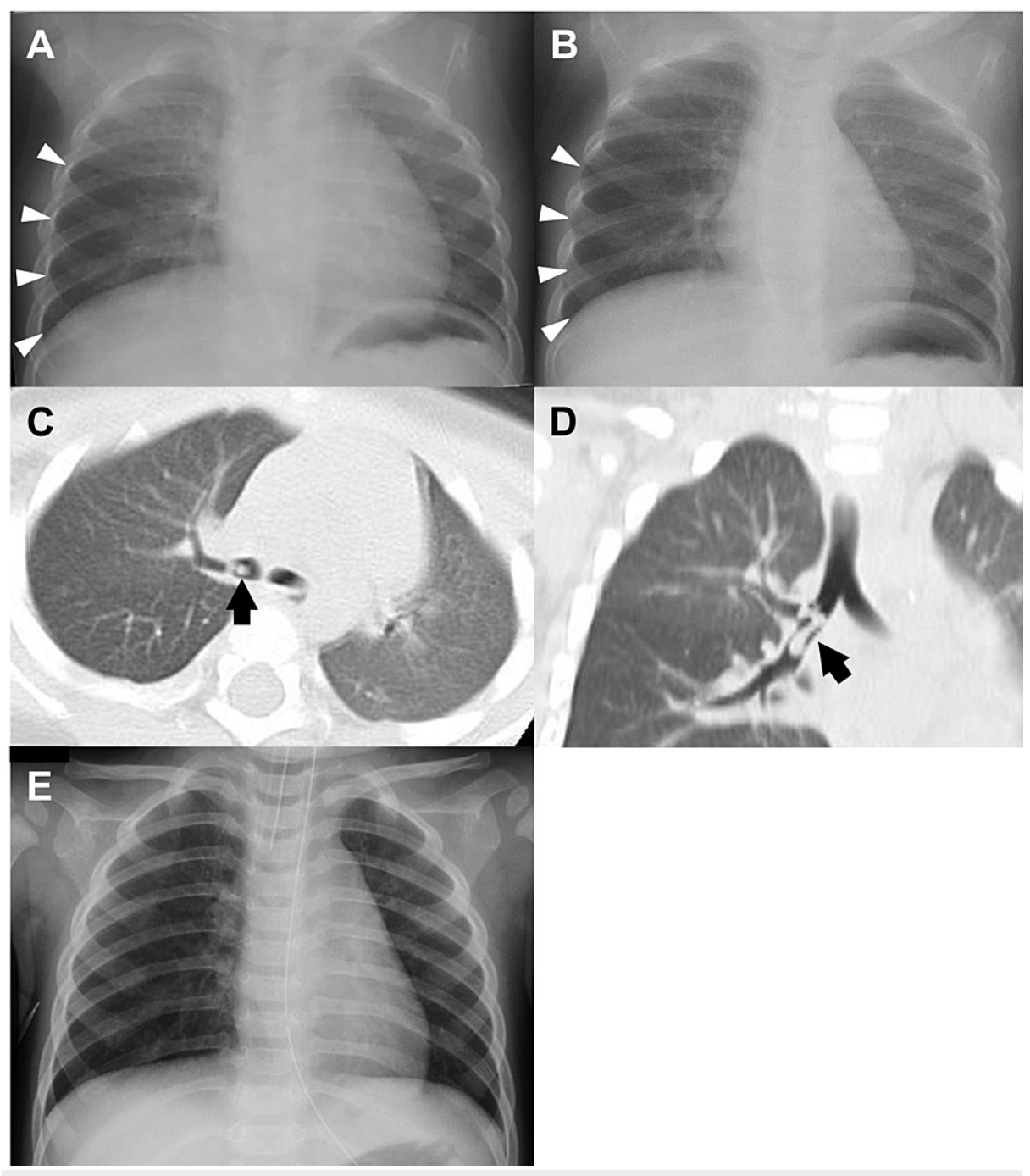

FIGURE 1: CXR showing slight emphysema of the right lower lung.

(A) Expiratory phase. (B) Inspiratory phase. (C) Axial CT showing a plastic foreign body at the right inferior lobar bronchus (black arrow). (D) Coronal CT showing the seven-shaped foreign body (black arrow). (E) CXR, after removing the foreign body, in which partial emphysema disappeared.

CXR, chest X-ray; CT, computed tomography

\section{Case 2}

A 23-month-old boy presented with a slight cough and runny nose. He was taken to a secondary referral hospital and diagnosed with a viral airway infection. Oral dexamethasone, L-carbocysteine, and ambroxol were prescribed for two days. His symptoms improved, but CXR showed slight segmental emphysema in the left lower lobe. This finding led to a series of observations (Figures $2 A, 2 B$ ). Further history evaluation revealed that he accidentally inserted small plastic parts of a pen into his nostrils three months prior to presentation. Subsequent CT revealed the foreign body in the left lower lobar bronchus (Figures 2C, 2D). He was then transferred to our hospital and underwent emergent flexible bronchoscopy under general anesthesia. The foreign body was partially covered with granuloma; however, it was successfully removed via alligator forceps. The postoperative course was uneventful, and CXR on POD 1 was normal (Figure 2E). He was discharged on POD 4. 


\section{Cureus}

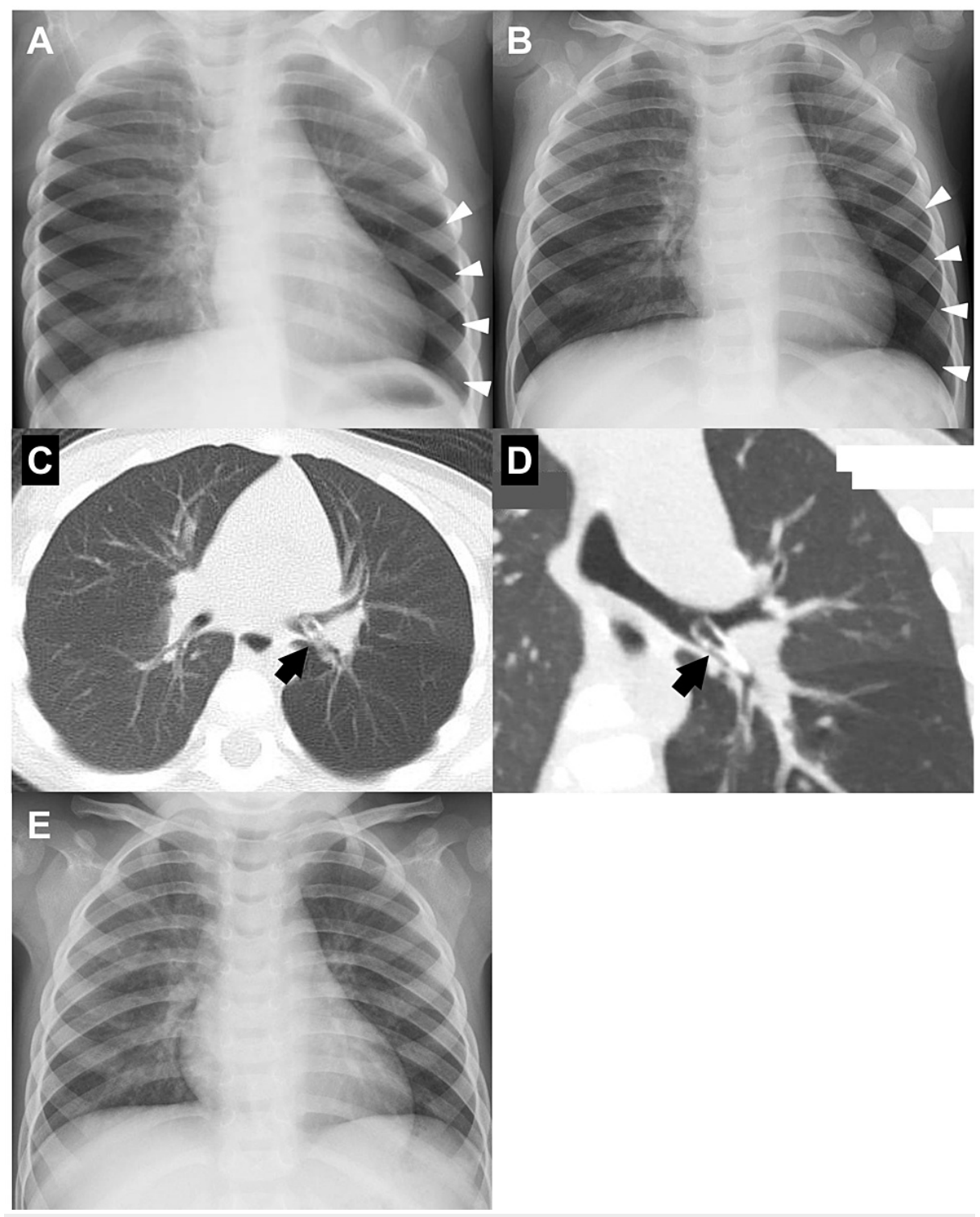

\section{FIGURE 2: CXR showing slight emphysema of the left lower lung.}

(A) CXR on the first day of visiting the hospital. (B) CXR four days later. (C) Axial CT showing a plastic foreign body at the left inferior lobar bronchus (black arrow). (D) Coronal CT showing the cranial foreign body (black arrow). (E) CXR after removing the foreign body, in which partial emphysema disappeared.

CXR, chest X-ray; CT, computed tomography

\section{Discussion}

FBA is problematic in the pediatric population, especially in younger age groups with narrow airways. Making the diagnosis is usually the main challenge, as the symptoms vary and are unspecific. Also, the act is often unwitnessed $[3,5,6]$. Additionally, the majority of foreign bodies are radiolucent, making the diagnosis even more difficult $[8]$.

In the diagnostic evaluation of FBA, stepwise methods are recommended in combination with taking a careful history, physical examination, and radiological investigations [10]. Among radiological imaging modalities, CT has a high sensitivity and specificity to detect most radiolucent foreign bodies [11-13]. In contrast, some reports prioritize direct bronchoscopy over radiological detections, as CT has a limited level of detection $[9,14]$. Since both of these investigations are invasive, candidates should be carefully selected.

CXR is a traditional non-invasive imaging modality that is widely available and affordable. Although the significance of CXR is still controversial in the diagnostic evaluation of FBA, it can be an important predictor $[4,5,8,9,11,12,15-17]$. In the present cases, history and slight symptoms were unspecific clues of FBA.

However, CXR showed partial emphysema without a significant mediastinal shift in the unilateral lung. This 
image led to further CT evaluation, which confirmed the presence of a lobar bronchial foreign body. In the initial evaluation, CXR played a significant role in predicting FBA in children.

In the face of unclear evidence of FBA, clinicians should place careful attention on CXR findings, especially unilateral partial emphysema, as shown in these cases. If possible, CXR should be taken in both inspiration and expiration in order to provide more significant information [7]. However, in younger children or infants, the timing of CXR is quite challenging because of a lack of following order. Therefore, it would be beneficial to continue follow-up of those patients, checking the symptoms and CXR repeatedly. Furthermore, recent reports showed the new tool to evaluate radiodensity on CXR, which has been expected to be useful in the diagnosis of FBA $[18,19]$.

To the best of our knowledge, this is the first case report to demonstrate typical images of partial emphysema without significant mediastinal shift caused by FBA, which would be helpful to clinicians.

\section{Conclusions}

We reported two cases of unilateral lobar emphysema on CXR, which is key to confirming FBA. Although CT or bronchoscopy would have been decisive in detecting FBA, performing and carefully interpreting a CXR is still important for recognizing the presence of a foreign body.

\section{Additional Information \\ Disclosures}

Human subjects: Consent was obtained or waived by all participants in this study. Shizuoka Children's Hospital Ethics Committee issued approval 2020014. Conflicts of interest: In compliance with the ICMJE uniform disclosure form, all authors declare the following: Payment/services info: All authors have declared that no financial support was received from any organization for the submitted work. Financial relationships: All authors have declared that they have no financial relationships at present or within the previous three years with any organizations that might have an interest in the submitted work. Other relationships: All authors have declared that there are no other relationships or activities that could appear to have influenced the submitted work.

\section{References}

1. Baharloo F, Veyckemans F, Francis C, Biettlot MP, Rodenstein DO: Tracheobronchial foreign bodies: presentation and management in children and adults. Chest. 1999, 115:1357-62. 10.1378/chest.115.5.1357

2. $\mathrm{Xu} \mathrm{B}, \mathrm{Wu} \mathrm{L}$, Jin Z, et al.: Residual airway foreign bodies in children who underwent rigid bronchoscopy . Int J Pediatr Otorhinolaryngol. 2019, 118:170-6. 10.1016/j.ijporl.2019.01.007

3. Karakoc F, Cakir E, Ersu R, et al.: Late diagnosis of foreign body aspiration in children with chronic respiratory symptoms. Int J Pediatr Otorhinolaryngol. 2007, 71:241-6. 10.1016/j.ijporl.2006.10.006

4. Wu Y, Dai J, Wang G, Li Y, Li H, Wu C, Wei G: Delayed diagnosis and surgical treatment of bronchial foreign body in children. J Pediatr Surg. 2020, 55:1860-5. 10.1016/j.jpedsurg.2019.10.052

5. Heyer CM, Bollmeier ME, Rossler L, Nuesslein TG, Stephan V, Bauer TT, Rieger CH: Evaluation of clinical, radiologic, and laboratory prebronchoscopy findings in children with suspected foreign body aspiration. J Pediatr Surg. 2006, 41:1882-8. 10.1016/j.jpedsurg.2006.06.016

6. Friedman EM: Tracheobronchial foreign bodies. Otolaryngol Clin North Am. 2000, 33:179-85. 10.1016/s0030-6665(05)70214-0

7. Griffiths DM, Freeman NV: Expiratory chest $x$ ray examination in the diagnosis of inhaled foreign bodies . Br Med J (Clin Res Ed). 1984, 288:1074-5. 10.1136/bmj.288.6423.1074

8. Tokar B, Ozkan R, Ilhan H: Tracheobronchial foreign bodies in children: importance of accurate history and plain chest radiography in delayed presentation. Clin Radiol. 2004, 59:609-15. 10.1016/j.crad.2004.01.006

9. Baram A, Sherzad H, Saeed S, Kakamad FH, Hamawandi AMH: Tracheobronchial foreign bodies in children: the role of emergency rigid bronchoscopy. Glob Pediatr Health. 2017, 4:2333794X17743663. 10.1177/2333794X17743663

10. Salih AM, Alfaki M, Alam-Elhuda DM: Airway foreign bodies: a critical review for a common pediatric emergency. World J Emerg Med. 2016, 7:5-12. 10.5847/wjem.j.1920-8642.2016.01.001

11. Bai W, Zhou X, Gao X, Shao C, Califano JA, Ha PK: Value of chest CT in the diagnosis and management of tracheobronchial foreign bodies. Pediatr Int. 2011, 53:515-8. 10.1111/j.1442-200X.2010.03299.X

12. Cavel O, Bergeron M, Garel L, Arcand P, Froehlich P: Questioning the legitimacy of rigid bronchoscopy as a tool for establishing the diagnosis of a bronchial foreign body. Int J Pediatr Otorhinolaryngol. 2012, 76:194201. 10.1016/j.ijporl.2011.11.002

13. Pitiot V, Grall M, Ploin D, Truy E, Ayari Khalfallah S: The use of CT-scan in foreign body aspiration in children: a 6 years' experience. Int J Pediatr Otorhinolaryngol. 2017, 102:169-73. 10.1016/j.ijporl.2017.08.036

14. Qiu W, Wu L, Chen Z: Foreign body aspiration in children with negative multi-detector computed tomography results: own experience during 2011-2018. Int J Pediatr Otorhinolaryngol. 2019, 124:90-3. 10.1016/j.ijporl.2019.05.031

15. Reid A, Hinton-Bayre A, Vijayasekaran S, Herbert H: Ten years of paediatric airway foreign bodies in Western Australia. Int J Pediatr Otorhinolaryngol. 2020, 129:109760. 10.1016/j.ijporl.2019.109760

16. Loo CM, Hsu AA, Eng P, Ong YY: Case series of bronchoscopic removal of tracheobronchial foreign body in six adults. Ann Acad Med Singap. 1998, 27:849-53. 


\section{Cureus}

17. Cohen S, Avital A, Godfrey S, Gross M, Kerem E, Springer C: Suspected foreign body inhalation in children: what are the indications for bronchoscopy?. J Pediatr. 2009, 155:276-80. 10.1016/j.jpeds.2009.02.040

18. Song ES, Han DK, Cho HJ, Jeong IS, Yoon N, Ma JS, Cho YK: Radiodensity on serial chest X-rays for the diagnosis of foreign body aspiration in children. Indian Pediatr. 2015, 52:663-7. 10.1007/s13312-015-0693-Z

19. Caliskan E, Aliyev S, Habibi HA, Bayramoglu Z, Yilmaz R, Adaletli I: Utility of lung radiodensity ratios in diagnosis of radiolucent foreign body aspiration in children: a practical approach. Clin Imaging. 2019, 54:178-82. 10.1016/j.clinimag.2018.03.005 